\title{
Low-dose UVB irradiation stimulates matrix metalloproteinase-1 expression via a BLT2-linked pathway in HaCaT cells
}

\author{
Cheolmin Kim, Ho-Cheol Ryu \\ and Jae-Hong Kim ${ }^{1}$ \\ College of Life Sciences and Biotechnology \\ Korea University \\ Seoul 136-701, Korea \\ ${ }^{1}$ Corresponding author: Tel, 82-2-3290-3452; \\ Fax, 82-2-923-0851; E-mail, jhongkim@korea.ac.kr \\ DOI 10.3858/emm.2010.42.12.086
}

Accepted 20 October 2010

Available Online 22 October 2010

Abbreviations: 12(S)-HETE, 12(S)-hydroxyeicosatetraenoic acid; 12LO, 12-lipoxygenase; BLT2, a low-affinity leukotriene $B_{4}$ receptor; $\mathrm{LTB}_{4}$, leukotriene $\mathrm{B}_{4} ; \mathrm{MMP}-1$, matrix metalloproteinase-1; Nox1, NADPH oxidase 1; UVB, ultraviolet $B$

\begin{abstract}
Skin exposure to low-dose ultraviolet B (UVB) light up-regulates the expression of matrix metalloproteinase-1 (MMP-1), thus contributing to premature skin aging (photo-aging). Although cyclooxygenase-2 (COX2) and its product, prostaglandin $E_{2}\left(P E_{2}\right)$, have been associated with UVB-induced signaling to MMP expression, very little are known about the roles of lipoxygenases and their products, especially leukotriene $\mathrm{B}_{4}\left(\mathrm{LTB}_{4}\right)$ and $12(\mathrm{~S})$-hydroxyeicosatetraenoic acid (12(S)-HETE), in MMP-1 expression in skin keratinocytes. In the present study, we demonstrate that BLT2, a cell surface receptor for $\mathrm{LTB}_{4}$ and 12(S)-HETE, plays a critical role in UVB-mediated MMP-1 upregulation in human $\mathrm{HaCaT}$ keratinocytes. Moreover, our results demonstrated that BLT2-mediated MMP-1 upregulation occurs through a signaling pathway dependent on reactive oxygen species (ROS) production and the subsequent stimulation of ERK. Blockage of BLT2 via siRNA knockdown or with the BLT2-antagonist LY255283 completely abolished the up-regulated expression of MMP-1 induced by low-dose UVB irradiation. Finally, when $\mathrm{HaCaT}$ cells were transiently transfected with a BLT2 expression plasmid, MMP-1 expression was significantly enhanced, along with ERK phosphorylation, suggesting that BLT2 overexpression alone is sufficient for MMP-1 up-regulation. Together, our results suggest that the BLT2-ROS-
\end{abstract}

ERK-linked cascade is a novel signaling mechanism for MMP-1 upregulation in low-dose UVB- irradiated keratinocytes and thus potentially contributes to photo-aging.

Keywords: 12-hydroxy-5,8,10,14-eicosatetraenoic acid; leukotriene $\mathrm{B}_{4}$; $\mathrm{LTB}_{4} \mathrm{R} 2$ protein, human; matrix metalloproteinase 1 ; reactive oxygen species; skin aging; ultraviolet rays

\section{Introduction}

Ultraviolet B (UVB) light is absorbed into the epidermis and causes various skin disorders. Whereas high-dose UVB irradiation causes apoptotic (e.g., sunburn) (Faustin and Reed, 2008; Yoshizumi et al., 2008; Ryu et al., 2010) or cancerous (Matsumura and Ananthaswamy, 2004; El-Abaseri et al., 2005) phenotypes in skin, low-dose UVB exposure has been suggested to accelerate skin aging or photo-aging, which includes major phenotypes such as wrinkles and sagging (Jenkins, 2002). The expression of matrix metalloproteinases (MMPs), which degrade cutaneous proteins (e.g., collagen and gelatin), is upregulated by low-dose UVB irradiation, resulting in a loss of elasticity and increased wrinkle formation in dermal tissue (Brenneisen et al., 2002).

MMPs can be classified into several groups including collagenase, gelatinase, stromelysin and membrane-type MMPs. MMP expression in normal tissues appears to be tightly regulated and is induced by various factors such as cytokines, growth factors, tumor promoters, oxidative stresses, heat-shock, and UV irradiation (Matrisian, 1994; Sternlicht and Werb, 2001). Collagen is a major structural component of the skin and maintenance of its level is important for skin elasticity. Among the various MMPs, MMP-1 is one of the important players in UVB-induced skin aging via collagen degradation (Kim et al., 2005, 2009). UV exposure leads to inflammatory responses in dermal skin (Hruza and Pentland, 1993; Katiyar et al., 1999; Pillai et al., 2005). Additionally, pro-inflammatory lipid mediators, such as prostaglandins (PGs) and leukotrienes (LTs), are inducibly synthesized following UV irradiation of dermal skin (Isoherranen et al., 1999; Yan et al., 2006). While many reports indicate that cyclooxygenase-2 
(COX-2) and its product, prostaglandin $\mathrm{E}_{2}\left(\mathrm{PGE}_{2}\right)$, are associated with UVB irradiation-induced MMP expression in human skin (Isoherranen et al., 1999; Seo et al., 2003), little is known about the roles of lipoxygenase (LO)-derived lipid mediators in mediating MMP upregulation in UVB-irradiated skin. Recently, several groups have reported that the induced activity and/or expression of 5-lipoxygenase (5-LO) results in remarkable elevation of leukotriene $\mathrm{B}_{4}\left(\mathrm{LTB}_{4}\right)$ (Yan et al., 2006) and the induction of 12-lipoxygenase (12-LO), which is followed by elevated synthesis of 12(S)-hydroxyeicosatetraenoic acid (12(S)-HETE) (Rhodes et al., 2009) in the skin after UV irradiation.

$\mathrm{LTB}_{4}$ is derived from arachidonic acid (AA) by the sequential actions of cytosolic phospholipase $A_{2}\left(C P L A_{2}\right), 5-L O$, and leukotriene $A_{4}\left(L_{T A}\right)$ hydrolase and is recognized by two kinds of $\mathrm{LTB}_{4}$ receptors, BLT1 and BLT2. Unlike BLT1, BLT2 is ubiquitously expressed in human tissues and has broad substrate specificity for several eicosanoids, including 12-HHT, 12(S)-HETE, 12(S)-HPETE, and 15(S)-HETE, as well as LTB $_{4}$ (Tager and Luster, 2003). Though it has been reported that BLT2 is highly expressed in mouse skin and causes sunburn damage in response to high-dose UVB irradiation or the itch-associated scratching of mouse skin in response to products of 12-LO (lizuka et al., 2005; Kim et al., 2008; Ryu et al., 2010), the exact function of BLT2 in skin has yet to be determined. In particular, no clear physiological role of BLT2 has been characterized in low dose UVB-induced photo-aging, especially in relation to MMP-1 expression.

Therefore, the aim of the present study was to investigate whether BLT2 plays a role in low-dose UVB irradiation-induced MMP-1 upregulation. We found that low-dose UVB irradiation significantly upregulated MMP-1 expression in human keratinocyte $\mathrm{HaCaT}$ cells through a pathway dependent on BLT2 (a receptor for $\mathrm{LTB}_{4}$ and 12(S)HETE). We also demonstrated that in UVB-irradiated HaCaT cells, BLT2 mediates MMP-1 upregulation through a signaling pathway dependent on reactive oxygen species (ROS) production and the subsequent stimulation of extracellularregulated kinase (ERK), two well-characterized mediators of UVB-induced MMP-1 expression (Whitmarsh and Davis, 1996; Wertz et al., 2004; Kim et al., 2005; Shin et al., 2008; Yang et al., 2009). In agreement with the proposed role of BLT2 as a mediator of UVB-induced MMP-1 expression, we observed that BLT2 overexpression markedly enhanced MMP-1 expression and ERK phosphorylation. Taken together, our results demonstrate that a BLT2-ROS-ERK-linked pathway
A

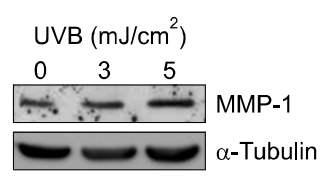

B

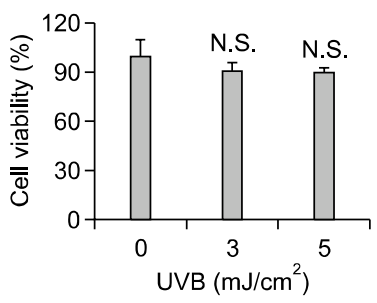

Figure 1. Low-dose UVB irradiation increases MMP-1 expression in HaCaT cells. (A) and (B), HaCaT cells were starved in serum-free DMEM for $24 \mathrm{~h}$ and then the cells were irradiated with increasing doses of UVB $\left(0,3\right.$, and $\left.5 \mathrm{~mJ} / \mathrm{cm}^{2}\right)$. (A) Levels of secreted MMP-1 in the culture medium were detected by western blotting $48 \mathrm{~h}$ after UVB irradiation; a-tubulin was used as a loading control. The results shown are representative of three independent experiments with similar results. (B) Cell viability was measured by the trypan blue exclusion assay $24 \mathrm{~h}$ after UVB irradiation. Data are the mean \pm S.D. of three independent experiments (N.S., not significant).

plays a crucial role in low-dose UVB-induced MMP-1 expression in human keratinocytes and thus potentially contributes to skin photo-aging.

\section{Results}

\section{Low-dose UVB irradiation up-regulates MMP-1 expression in $\mathrm{HaCaT}$ cells}

To examine the possible role of BLT2 in MMP-1 expression in response to UVB irradiation in HaCaT cells, we first tested whether MMP-1 expression is upregulated by low-dose UVB irradiation. To answer this question, we carried out western blotting using an equal volume of conditioned medium from UVB-irradiated cells $(0,3$, and $5 \mathrm{~mJ} / \mathrm{cm}^{2} ; 48 \mathrm{~h}$ irradiation) and observed that MMP-1 expression was significantly increased by UVB exposure (Figure 1A). We performed trypan blue exclusion assay to measure cell viability and did not observe any cytotoxicity to cells under the low-dose UVB irradiation conditions $\left(\leq 5 \mathrm{~mJ} / \mathrm{cm}^{2}\right.$, see Methods for details) (Figure 1B). However, significant cytotoxicity was detected with high-dose UVB irradiation $\left(\leq 10 \mathrm{~mJ} / \mathrm{cm}^{2}\right)$ (data not shown). Together, these results suggest that a low dose (5 $\mathrm{mJ} / \mathrm{cm}^{2}$ ) of UVB irradiation markedly increases MMP-1 expression in HaCaT keratinocytes without affecting cell viability.

\section{UVB-induced MMP-1 expression is regulated through a BLT2-cascade in HaCaT cells}

Next, we tested whether BLT2 and its ligands, $\mathrm{LTB}_{4}$ and 12(S)-HETE, play a role in low-dose UVBinduced MMP-1 expression in HaCaT cells. Ini- 
A

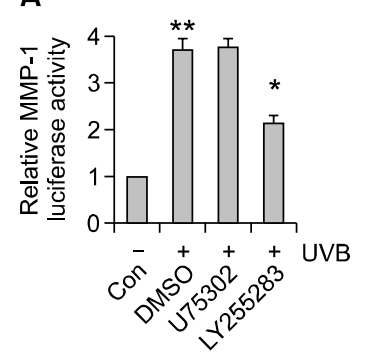

C

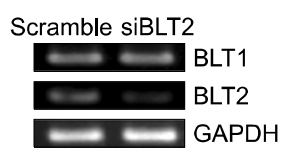

D

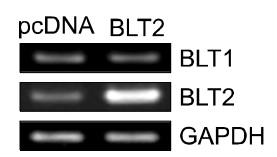

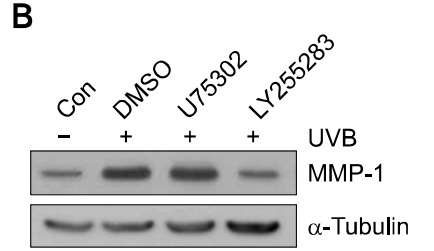
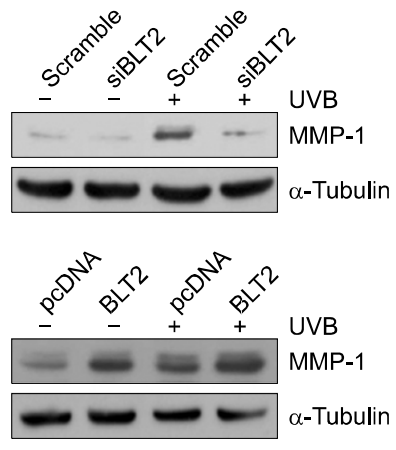

Figure 2. Low-dose UVB-induced MMP-1 expression is dependent on BLT2 in HaCaT cells. (A) HaCaT cells were transiently transfected with the MMP-1 reporter gene as described in Methods. The cells were starved in serum-free DMEM for $24 \mathrm{~h}$. The cells were then incubated for $1 \mathrm{~h}$ with $1 \mu \mathrm{M}$ U75302 or $10 \mu \mathrm{M}$ LY255283 prior to UVB irradiation. Protein extracts were assayed for luciferase activity $24 \mathrm{~h}$ after UVB irradiation $\left(5 \mathrm{~mJ} / \mathrm{cm}^{2}\right)$. Data are the mean \pm S.D. of three independent experiments ${ }^{*}, P<0.05$; $\left.{ }^{* *}, P<0.01\right)$. (B) HaCaT cells were starved in serum-free DMEM for $24 \mathrm{~h}$. The cells were then incubated for $1 \mathrm{~h}$ with $1 \mu \mathrm{M}$ U75302 or $10 \mu \mathrm{M}$ LY255283 prior to UVB irradiation. Levels of secreted MMP-1 in the culture medium were detected by western blotting $48 \mathrm{~h}$ after UVB irradiation $\left(5 \mathrm{~mJ} / \mathrm{cm}^{2}\right)$. (C and D) HaCaT cells were transiently transfected with $50 \mu \mathrm{M}$ BLT2-specific siRNA (C) or pcDNA-BLT2 (D) as described in Methods. The cells were then starved in serum-free DMEM for $24 \mathrm{~h}$ prior to UVB irradiation. Levels of secreted MMP-1 in the culture medium were detected by western blotting $48 \mathrm{~h}$ after UVB irradiation ( 5 $\left.\mathrm{mJ} / \mathrm{cm}^{2}\right) ; \alpha$-tubulin was used as a loading control. The results shown are representative of three independent experiments with similar results. To determine transfection efficiency, the total RNA was isolated, and the level of the BLT2 transcript was determined by semi-quantitative RT-PCR (C and D). The GAPDH level was used as a control.

tially, we examined the effect of a BLT2 antagonist (LY255283) on UVB-induced stimulation of MMP-1 promoter activity. As seen in Figure 2A, pretreatment with LY255283 remarkably suppressed UVB-stimulated MMP-1 promoter activity, whereas pretreatment with U75302, a BLT1 antagonist, had no effect. Similarly, western blotting analysis of the conditioned medium from UVB-irradiated cells showed that pretreatment with LY255283, but not U75302, clearly abolished the induced expression levels of MMP-1 (Figure 2B). Moreover, we observed that BLT2 knockdown with siRNA (siBLT2) completely suppressed UVB-induced MMP-1 expression (Figure 2C, right). These results suggest that $\mathrm{BLT} 2$ is crucial in mediating the low-dose UVB signaling of MMP-1 expression

in HaCaT cells. Under the siBLT2 knockdown experimental conditions, the levels of BLT2, but not BLT1, were specifically reduced (Figure 2C, left). Additionally, we could observe a similar inhibitory effect by LY255283, but not by U75302, on UVB-induced MMP-1 expression in primary keratinocytes (data not shown), suggesting a potential common role of BLT2 in mediating MMP-1 expression in keratinocytes. Finally, to further demonstrate the role of BLT2 in mediating UVBsignaling of MMP-1 expression, we examined whether BLT2 overexpression is sufficient for inducing the expression of MMP-1. Thus, we transiently transfected $\mathrm{HaCaT}$ cells with the pcDNABLT2 expression plasmid. Under these conditions, elevated BLT2 mRNA levels were detected by semiquantitative RT-PCR analysis (Figure 2D, left). We clearly observed greatly enhanced MMP-1 expression through BLT2 overexpression alone (lane 2) compared to the control (Figure 2D, right). With UVB irradiation, further enhancement of MMP-1 expression was detected (Figure 2D, right). Therefore, these results suggest that BLT2 plays a critical mediatory role in UVB-induced MMP-1 expression in $\mathrm{HaCaT}$ cells.

\section{UVB irradiation enhances the levels of BLT2 ligands and their synthesizing enzymes in $\mathrm{HaCaT}$ cells}

To further elucidate the mediatory role of BLT2 in UVB-induced MMP-1 expression, we examined whether levels of the BLT2 ligands $\mathrm{LTB}_{4}$ and 12(S)-HETE are also elevated in UVB-irradiated HaCaT cells. To explore this question, we performed specific enzyme-linked immunosorbent assays (ELISAs) to measure the levels of these ligands following UVB irradiation. Figure $3 A$ shows that low-dose UVB irradiation greatly increased the production levels of $\mathrm{LTB}_{4}$ and 12(S)-HETE. Next, we assessed the expression levels of 5 - and 12-LO, which are enzymes responsible for the synthesis of $\mathrm{LTB}_{4}$ or $12(\mathrm{~S})-\mathrm{HETE}$, respectively. Figure $3 \mathrm{~B}$ shows that 5 - and 12-LO levels were also significantly upregulated following UVB irradiation. Blockage of $\mathrm{LTB}_{4}$ and 12(S)-HETE synthesis with MK886 (5-LO/5-LO activating protein inhibitor) and Baicalein (12-LO inhibitor), respectively, was shown to diminish UVB-induced MMP-1 expression (Figure 3C). However, the addition of $\mathrm{LTB}_{4}$ or 12(S)-HETE to the inhibitor-treated cells restored UVB-induced MMP-1 expression (Figure $3 C)$. These results suggest that BLT2 signaling, along with augmented synthesis of its ligands $\mathrm{LTB}_{4}$ and 12(S)-HETE, play roles in mediating MMP-1 expression in response to UVB irradiation in $\mathrm{HaCaT}$ cells. 
A

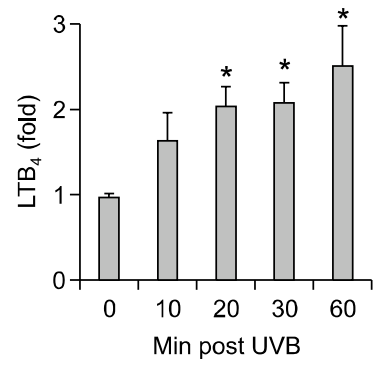

B

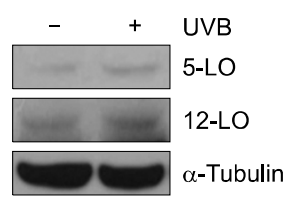

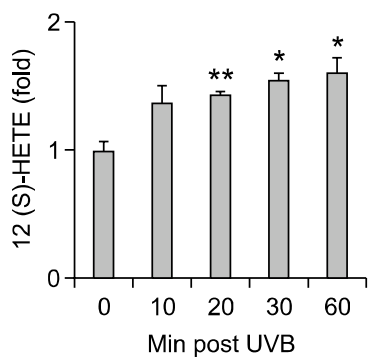
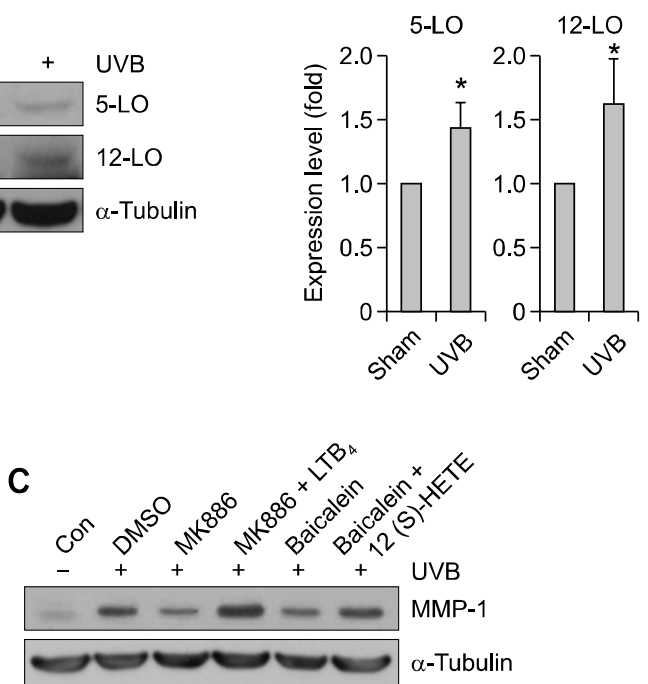

Figure 3. Low-dose UVB irradiation enhances the levels of BLT2 ligands and their synthesizing enzymes (5-LO and 12-LO) in HaCaT cells. (A) $\mathrm{HaCaT}$ cells were starved in serum-free DMEM for $24 \mathrm{~h}$ prior to UVB irradiation. The level of secreted $\mathrm{LTB}_{4}$ or 12(S)-HETE in the culture medium was determined by specific ELISAs at the indicated times $(0,10$, 20,30 , and $60 \mathrm{~min}$ ) after UVB irradiation. Data are the mean \pm S.D. ( ${ }^{*}$, $P<0.05$; ${ }^{* *}, P<0.01 ; n=5$ )(see Methods for details). (B) Proteins were extracted from cells irradiated with UVB $\left(5 \mathrm{~mJ} / \mathrm{cm}^{2}, 30 \mathrm{~min}\right)$ and the protein levels were western blotted using antibodies against 5- or 12-LO; $\alpha$-tubulin was used as a loading control. The results shown are representative of three independent experiments with similar results. The expression levels of 5- and 12-LO were assessed using Student's t-test and expressed as mean \pm S.D. $\left({ }^{*}, P<0.05\right)$. (C) The cells were incubated for $1 \mathrm{~h}$ with $10 \mu \mathrm{M}$ MK886 or $20 \mu \mathrm{M}$ baicalein prior to UVB irradiation. Levels of secreted MMP-1 in the culture medium were detected by western blotting $48 \mathrm{~h}$ after UVB irradiation $\left(5 \mathrm{~mJ} / \mathrm{cm}^{2}\right) ; \alpha$-tubulin was used as a loading control.

\section{Low-dose UVB-induced MMP-1 expression is dependent on the BLT2-ROS-linked cascade}

Accumulating evidence suggests that elevated ROS have mediatory roles in the expression of MMPs induced by UVB (Fisher et al., 1996; Whitmarsh and Davis, 1996; Brenneisen et al., 2002; Vayalil et al., 2004). Additionally, we previously reported that an $\mathrm{LTB}_{4}-\mathrm{BLT} 2$ cascade is associated with the generation of ROS, which cause various cellular responses (Woo et al., 2002; Choi et al., 2008; Ryu et al., 2010). Therefore, we
A

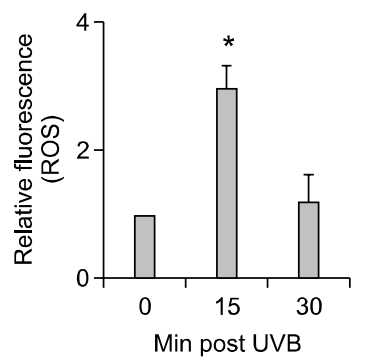

B

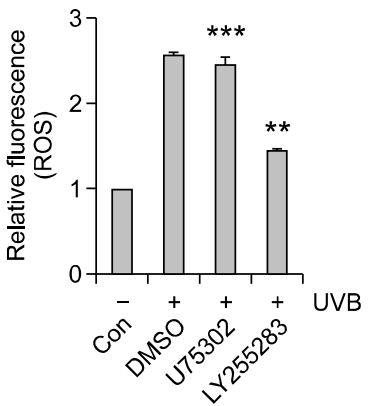

C
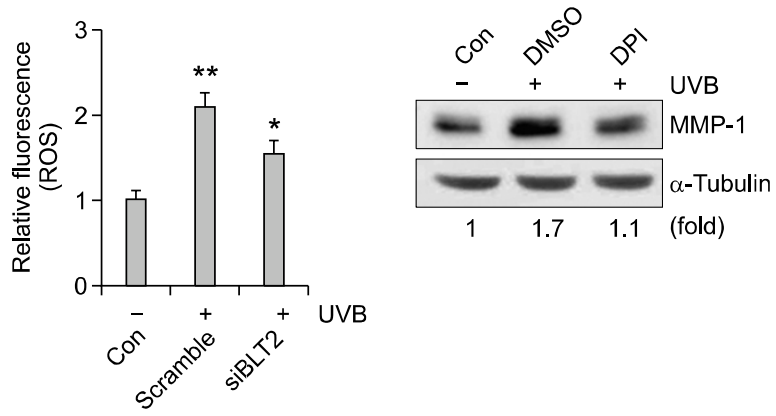

Figure 4. Low-dose UVB-induced MMP-1 expression is dependent on the BLT2-ROS-linked cascade. HaCaT cells were starved in serum-free DMEM for $24 \mathrm{~h}$ prior to UVB irradiation. The cells were incubated with 20 $\mu \mathrm{M}$ 2', 7'-dichlorofluorescein diacetate (DCFDA), an $\mathrm{H}_{2} \mathrm{O}_{2}$-sensitive fluorophore, for $20 \mathrm{~min}$ in the dark. Intracellular ROS levels were monitored by FACS analysis of DCF fluorescence after UVB irradiation $\left(5 \mathrm{~mJ} / \mathrm{cm}^{2}\right)$. (A) HaCaT cells were irradiated for the indicated times $(0,15$, and 30 $\mathrm{min})$ before ROS measurement. Also, cells were pretreated with inhibitors (1 $\mu \mathrm{M}$ U75302 or $10 \mu \mathrm{M}$ LY255283) (B) for $1 \mathrm{~h}$ or transfected with $50 \mu \mathrm{M}$ scrambled siRNA or siBLT2 for $24 \mathrm{~h}(\mathrm{C})$ prior to UVB irradiation. Then, the ROS levels were measured. All data are the mean \pm S.D. of three independent experiments $\left({ }^{*}, P<0.05\right.$; ${ }^{* *}, P<0.01$; ${ }^{* * *}, P<0.001$ ). (D) The cells were incubated with $0.1 \mu \mathrm{M} D P I$ for $1 \mathrm{~h}$ prior to UVB irradiation. Levels of secreted MMP-1 in the culture medium were detected by western blotting $48 \mathrm{~h}$ after UVB irradiation $\left(5 \mathrm{~mJ} / \mathrm{cm}^{2}\right)$; $\alpha$-tubulin was used as a loading control. The results shown are representative of three independent experiments with similar results.

suspected that BLT2 may play a role in the generation of ROS triggered by low-dose UVB irradiation. To test this suspicion, ROS levels were monitored by FACS analysis at 0,15 , and $30 \mathrm{~min}$ after UVB irradiation $\left(5 \mathrm{~mJ} / \mathrm{cm}^{2}\right)$. Similar to previous reports, we observed that low-dose UVB irradiation rapidly increases ROS generation, reaching a maximum within $15 \mathrm{~min}$ (Figure 4A). Additionally, pretreatment with LY255283, but not U75302, significantly suppressed low-dose UVBinduced ROS generation (Figure 4B), suggesting a mediatory role for BLT2 in UVB-induced ROS generation. To further examine the role of BLT2 in ROS generation by low-dose UVB irradiation, we knocked down BLT2 with siBLT2 and similar 
A

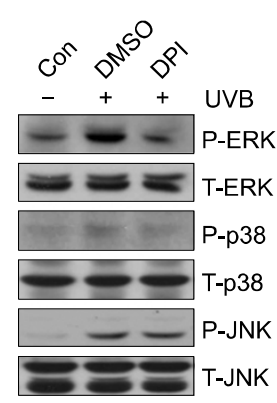

C
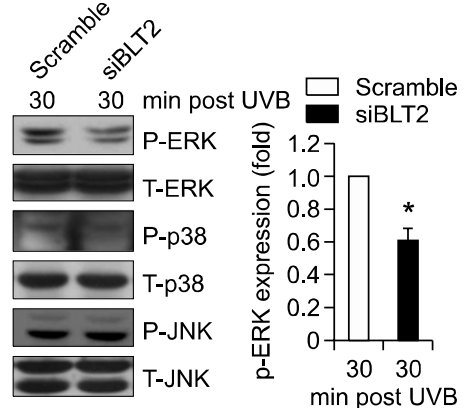

D
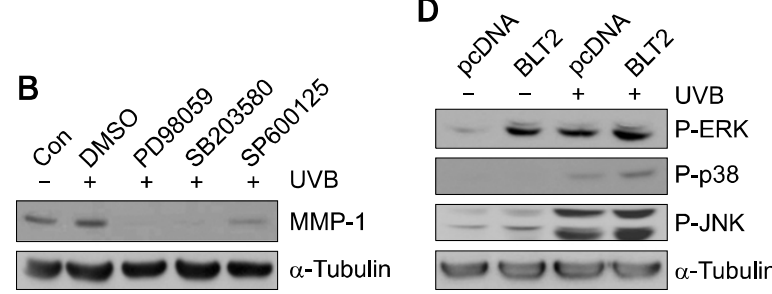

Figure 5. ERK phosphorylation lies downstream of the BLT2-ROS cascade in UVB signaling of MMP-1 expression in $\mathrm{HaCaT}$ cells. (A) After starvation for $24 \mathrm{~h}$ in serum-free DMEM, the cells were incubated with $0.1 \mu \mathrm{M}$ DPI for $1 \mathrm{~h}$ prior to UVB irradiation. Proteins were extracted, western-blotted, and hybridized with antibodies against MAPKs (p-ERK, $\mathrm{p}$-p38, and p-JNK) $30 \mathrm{~min}$ after UVB irradiation $\left(5 \mathrm{~mJ} / \mathrm{cm}^{2}\right)$. The results shown are representative of three independent experiments with similar results. (B) The cells were incubated with $20 \mu \mathrm{M}$ PD98059, SB203580, or SP600125 for $1 \mathrm{~h}$ prior to UVB irradiation. Levels of secreted MMP-1 in the culture medium were detected by western blotting $48 \mathrm{~h}$ after UVB irradiation $\left(5 \mathrm{~mJ} / \mathrm{cm}^{2}\right) ; \alpha$-tubulin was used as a loading control. The results shown are representative of three independent experiments with similar results. (C) The cells were transiently transfected with $50 \mu \mathrm{M}$ scrambled or BLT2-specific siRNA and then starved for $24 \mathrm{~h}$ in serum-free DMEM. Proteins were extracted and western blotted with antibodies against MAPKs (p-ERK, p-p38, and p-JNK) at $30 \mathrm{~min}$ after UVB irradiation. The levels of $p$-ERK were assessed using Student's t-test and expressed as mean \pm S.D. of three independent experiments ${ }^{*}, P<$ $0.05)$. (D) HaCaT cells were transiently transfected with pcDNA or pcDNA-BLT2 for $24 \mathrm{~h}$. After transfection, cells were starved in serum-free DMEM for $24 \mathrm{~h}$ prior to UVB irradiation. Proteins were extracted and western blotted with antibodies against MAPKs ( $p$-ERK, p-p38, and p-JNK) 30 min after UVB irradiation $\left(5 \mathrm{~mJ} / \mathrm{cm}^{2}\right) ; \alpha$-tubulin was used as a loading control. The results shown are representative of three independent experiments with similar results.

suppression of UVB-induced ROS generation was observed (Figure $4 \mathrm{C}$ ). This suggests that ROS generation occurs through a BLT2-dependent pathway in response to low-dose UVB irradiation. In agreement with previous reports, we observed that pretreatment with diphenylene iodonium (DPI), an inhibitor of flavoenzymes such as NADPH oxidase, attenuates UVB-induced MMP-1 expression (Figure 4D). When taken together, these results suggest that the BLT2-ROS cascade mediates UVB-induced MMP-1 expression.

\section{ERK phosphorylation lies downstream of the BLT2-ROS cascade in UVB signaling of MMP-1 in HaCaT cells}

Extracellular-regulated kinase (ERK) is stimulated by elevated ROS and activates downstream signaling involving AP-1, which results in MMP expression (Whitmarsh and Davis, 1996; Shin et al., 2008; Shim et al., 2009). ERK stimulation may therefore lie downstream of the BLT2-ROS cascade to mediate MMP-1 expression in UVB-irradiated $\mathrm{HaCaT}$ cells. To test this hypothesis, we determined whether ERK phosphorylation is affected by pretreatment with the antioxidant DPI or by BLT2 knockdown with siBLT2. In agreement with previous observations reporting that mitogen-activated protein kinases (MAPKs), including ERK, play roles in UV-induced MMP-1 expression (Brenneisen et al., 2002; Wertz et al., 2004; Kim et al., 2005; Shin et al., 2008; Shim et al., 2009; Yang et al., 2009), ERK phosphorylation was greatly enhanced by low-dose UVB irradiation in $\mathrm{HaCaT}$ cells (Figure 5A). Additionally, JNK and p38 kinase levels were also stimulated by UVB irradiation, although the levels of phosphorylation were not as great as those of ERK. Pretreatment of the cells with PD98059 (an ERK inhibitor), SB203580 (a p38 kinase inhibitor), or SP600125 (a JNK inhibitor) clearly diminished the upregulation of MMP-1 in UVB-irradiated HaCaT cells (Figure 5B), suggesting that MAPK stimulation plays a role in the upregulation of MMP-1 in response to low-dose UVB irradiation. Next, to assess if ERK (or other MAPKs) stimulation lies downstream of BLT2 in UVB-irradiated cells, we tested the effect of BLT2 knockdown on UVB-induced MAPK stimulation. As shown in Figure $5 \mathrm{C}$, BLT2 knockdown with siBLT2 clearly reduced ERK phosphorylation without affecting the phosphorylation of other MAPKs (i.e., JNK or p38 kinase). To further demonstrate the role of BLT2 in mediating UVB signaling of ERK phosphorylation, we examined whether BLT2 overexpression is sufficient for inducing ERK phosphorylation. We clearly observed greatly enhanced ERK phosphorylation by BLT2 overexpression alone (lane 2) compared to the control (Figure 5D). Additionally, further enhanced ERK phosphorylation was detected with UVB irradiation (Figure 5D). Taken together, these results suggest that ERK lies downstream of the BLT2-ROS cascade in the low-dose UVB-induced signaling pathway in $\mathrm{HaCaT}$ cells.

\section{Discussion}

In the present study, we demonstrated that a 
BLT2-linked pathway is associated with low-dose UVB-induced MMP-1 expression in keratinocytes. This conclusion is based on the following observations: (1) BLT2 blockage using a specific antagonist or siRNA reduces low-dose UVB irradiation-induced MMP-1 expression; (2) BLT2 overexpression in $\mathrm{HaCaT}$ cells is sufficient to cause significant up-regulation of MMP-1 expression; (3) levels of both BLT2 ligands $\left(\mathrm{LTB}_{4}\right.$ and 12(S)-HETE) and the synthesizing enzymes for those ligands (5-LO and 12-LO, respectively) are considerably increased in response to UVB irradiation in $\mathrm{HaCaT}$ cells; and (4) blockage of the synthesis of BLT2 ligands with MK886 (an inhibitor of 5-LO) or Baicalein (an inhibitor of 12-LO) remarkably diminished UVB-induced MMP-1 expression. However the addition of $\mathrm{LTB}_{4}$ or $12(\mathrm{~S})$ HETE to inhibitor-treated cells restored UVBinduced MMP-1 expression.

Additionally, we provide evidence suggesting that BLT2-mediated MMP-1 upregulation occurs through a signaling pathway dependent on ROS production and the subsequent stimulation of ERK. Consistent with our observations, ROS are known to mediate low-dose UVB-induced MMP expression (Brenneisen et al., 2002; Vayalil et al., 2004; Wertz et al., 2004). In particular, the importance of ROS in photo-aging is underlined by extensive evidence that exposure to antioxidants decreases UVB-induced MMP expression in dermal skin cells (Kang et al., 2003; Vayalil et al., 2004; Wertz et al., 2004; Zaid et al., 2007). However, neither the source of ROS generated in response to low-dose UVB irradiation nor the pathway by which they are generated in the skin has been clearly understood. Among the various sources of ROS, NADPH oxidase (Nox) is a major regulator of MMP-1 expression (Shin et al., 2008). Also, we recently reported that the BLT2 cascade mediates ROS generation through NADPH oxidase 1 (Nox1) (Ryu et al., 2010). We previously observed that BLT2 overexpression induces enhanced Nox1 mRNA expression and ROS generation in $\mathrm{HaCaT}$ cells (Ryu et al., 2010). Based on these observations, we suspect that Nox, possibly Nox1, is associated with BLT2-mediated ROS generation in response to low-dose UVB in HaCaT cells. In support of this idea, we observed that UVB-induced MMP-1 expression was significantly suppressed by pretreatment with DPI (Figure 4D). Nonetheless, the exact mechanism by which BLT2 mediates ROS generation in response to low-dose UVB irradiation requires further examination.

Moreover, in the present study, our results suggest that ERK stimulation lies downstream of the BLT2-ROS cascade (Figure 5) that mediates
MMP-1 expression in UVB-irradiated HaCaT cells. In fact, our previous report showed that the BLT2-linked pathway regulates the high-dose UVBinduced sunburn response through signaling via the BLT2-Nox1-p38/JNK pathway (Ryu et al., 2010). Thus, although sunburn and photo-aging responses appear to share a common signaling mechanism, the downstream MAPKs involved in each response may be different. Whereas the sunburn response (induced by high-dose UVB) is likely to mainly be mediated through a pathway dependent on p38 kinase/JNK (Ryu et al., 2010), MMP-1 upregulation (and thus the photo-aging response triggered by low-dose UVB) is largely dependent on ERK. In support of the suggested role of ERK in photo-aging, but not sunburn apoptosis, ERK activation was shown to act as a survival signal in keratinocytes (He et al., 2004; Lee et al., 2005). Under our experimental conditions, the transient overexpression of BLT2 in $\mathrm{HaCaT}$ cells leads to remarkably enhanced ERK phosphorylation and MMP-1 upregulation (Figure $5 \mathrm{D}$ and Figure 2D), which again supports the close association of BLT2 with ERK stimulation. Similarly, our previous reports also show that ERK is activated by the BLT2 ligand (Woo et al., 2002, 2003). Nonetheless, we cannot exclude the possible contributory roles of p38 kinase or JNK in BLT2-mediated MMP-1 upregulation signaling. Although we suspect that ERK largely mediates the low-dose UVB-BLT2 pathway to upregulate MMP-1, other MAP kinases (e.g., p38 kinase and JNK) may play roles in the pathway, albeit to a much lesser extent.

MMP-1 is transcriptionally regulated by $N F-\kappa B$ and AP-1, which are activated by MAPKs (Fisher et al., 1996; Kim et al., 2005) or ROS (Garmyn and Degreef, 1997; Saliou et al., 1999). Consistent with this finding, we observed that pretreatment with the BLT2 antagonist LY255283 suppresses NF- $\mathrm{B} /$ AP-1 transcriptional activity, whereas the BLT1 antagonist had no effect (data not shown). Thus, we speculate that NF- $\mathrm{B} / \mathrm{AP}-1$ activation may lie downstream of the BLT2-linked pathway in UVBinduced MMP-1 upregulation in $\mathrm{HaCaT}$ cells. However, the detailed mechanism by which NF$\kappa \mathrm{B} / \mathrm{AP}-1$ activation contributes to the low-dose UVB-BLT2-linked pathway of MMP-1 upregulation must be further elucidated.

In summary, we demonstrated that BLT2 mediates low-dose UVB irradiation-induced MMP-1 expression in $\mathrm{HaCaT}$ cells. Additionally, we demonstrated that BLT2 mediates MMP-1 upregulation through a signaling pathway dependent on ROS production and the subsequent stimulation of ERK in UVB-irradiated HaCaT cells. Based on our findings, we propose that the BLT2-ROS-ERK- 
linked pathway may potentially contribute to photo-aging and that this pathway could be an effective target for attenuating photo-aging in skin.

\section{Methods}

\section{Cell culture and chemicals}

The immortalized human skin keratinocyte $\mathrm{HaCaT}$ cell line was maintained in Dulbecco's modified Eagle's medium (DMEM) containing $10 \%$ fetal bovine serum (FBS) in a $37^{\circ} \mathrm{C}, 5 \% \mathrm{CO}_{2}$ incubator. The cells were passaged every 3-4 days when they reached $80-90 \%$ confluency. All experiments were carried out in $60 \mathrm{~mm}$ dishes, and cells were grown to a confluent monolayer. The BLT2 antagonist LY255283, LTB $_{4}$ and 12(S)-HETE were purchased from the Cayman Chemical Company (Ann Arbor, MI). The BLT1 antagonist U75302 was from BioMol (Plymouth Meeting, PA). MK886, baicalein, and diphenyleneiodonium (DPI) were obtained from Calbiochem (La Jolla, CA). The monoclonal antibodies against MMP-1 were obtained from Calbiochem (La Jolla, CA). FBS and DMEM were from Gibco (Grand Island, NY). The polyclonal antibodies against MAP kinases (ERK, JNK, and p38 kinase) were obtained from Cell Signaling Technology (Danvers, MA). Anti-mouse and anti-rabbit secondary antibody horseradish peroxidase conjugates were purchased from Amersham Biosciences (Piscataway, NJ). The MAPK inhibitors PD98059, SB203580 and SP600125 were obtained from Calbiochem (San Diego, CA). All other chemicals were from standard sources and were of molecular biology grade or higher.

\section{UVB irradiation}

Cells were starved in serum-free DMEM for $24 \mathrm{~h}$. Before UVB irradiation, the medium was removed, the cells were washed with warm PBS, and $200 \mu$ of PBS was pipetted onto each dish to keep the cells wet. The cells were then irradiated with a UV crosslinker (Upland, CA), which incorporates $5 \times 8 \mathrm{~W}$ tubes that emit most of their energy within the UVB range with an emission peak at $302 \mathrm{~nm}$. The UVB dose was accurately calculated with a UVB meter. After irradiation, fresh serum-free DMEM was added to each dish.

\section{Cell viability}

Cell viability was assessed by the trypan blue exclusion assay $24 \mathrm{~h}$ after UVB irradiation. Whole cells were collected from the medium (detached cells) and culture dish (attached cells). A $10 \mu \mathrm{l}$ aliquot of the cells was then suspended in DMEM, and $10 \mu \mathrm{l}$ of $0.14 \%$ (w/v) trypan blue solution was added. Cells were counted using a hemocytometer under a light microscope. Cell viability is expressed as a percentage of the total cell population.

\section{Semiquantitative RT-PCR for BLT1 and BLT2}

Total cellular RNA was extracted from cells using an Easy
Blue RNA Extraction kit (Intron Biotechnology, Seongnam, Korea), dissolved in diethylpyrocarbonate-treated water, and quantified by UV scanning. The RNA was reverse transcribed using Moloney murine leukemia virus reverse transcriptase (Invitrogen, Carlsbad, CA); BLT1, BLT2, and glyceraldehyde 3-phosphate (GAPDH) transcripts were amplified using a PCR PreMix kit (Intron Biotechnology, Seongnam, Korea). cDNA from $2 \mu \mathrm{g}$ of total RNA was used for each PCR reaction. The primers for human BLT1 were 5'-TATGTCTGCGGAGTCAGCATGTACGC-3' (forward) and 5'-CCTGTAGCCGACGCCCTATGTCCG-3' (reverse). The PCR protocol for human BLT1 involved 30 cycles of denaturation at $94^{\circ} \mathrm{C}$ for $30 \mathrm{~s}$, annealing at $67^{\circ} \mathrm{C}$ for $30 \mathrm{~s}$, and elongation at $72^{\circ} \mathrm{C}$ for $30 \mathrm{~s}$, followed by an extension at $72^{\circ} \mathrm{C}$ for $10 \mathrm{~min}$. The primers for human BLT2 were 5'-TCTCATCGGGCATCACAGGT-3' (forward) and 5'-CCAAGCTCCACACCACGAAG-3' (reverse). The PCR protocol involved 35 cycles of denaturation at $94^{\circ} \mathrm{C}$ for $30 \mathrm{~s}$, annealing at $65^{\circ} \mathrm{C}$ for $30 \mathrm{~s}$, and elongation at $72^{\circ} \mathrm{C}$ for $30 \mathrm{~s}$, followed by extension at $72^{\circ} \mathrm{C}$ for $10 \mathrm{~min}$. The primers for GAPDH were 5'-CTGCACCACCAACTGCTTAGC-3' (forward) and 5'-CTTCACCACCTTCTTGATGTC-3' (reverse). The PCR protocol involved 22 cycles of denaturation at $94^{\circ} \mathrm{C}$ for $30 \mathrm{~s}$, annealing at $58^{\circ} \mathrm{C}$ for $30 \mathrm{~s}$, and elongation at $72^{\circ} \mathrm{C}$ for $30 \mathrm{~s}$, followed by extension at $72^{\circ} \mathrm{C}$ for $10 \mathrm{~min}$. The amplified PCR products were subjected to electrophoresis on $1.5 \%$ agarose gels, after which bands were visualized by ethidium bromide staining and photographed with a GelDoc system (BioRad, Hercules, CA). The specificity of all primers was confirmed by sequencing the PCR products. RNA extraction products were tested in control reverse transcription reactions and found to be free of DNA contamination.

\section{Western blotting}

To determine the levels of MMP-1 in culture, equal aliquots volume of conditioned culture medium, which were collected $48 \mathrm{~h}$ after UVB irradiation $\left(5 \mathrm{~mJ} / \mathrm{cm}^{2}\right)$ were separated by $10 \%$ SDS-PAGE and transferred to Hybond-P membranes (Amersham, NJ). The membranes were blocked and incubated with the primary antibody (MMP-1) and then with the horseradish peroxidase (HRP)-conjugated secondary antibody prior to development using an ECL kit (Amersham, NJ). The hybridized immunoblots were analyzed by autoradiography. Equal aliquots of whole cell lysates using the same volumes of RIPA lysis buffer were subjected to western blotting to measure a-tubulin as a loading control for MMP-1 PAGE. To assess MAPK phosphorylation, proteins were extracted using RIPA lysis buffer containing protease inhibitors 30 min after UVB irradiation.

\section{RNAi of BLT2}

The siBLT2 sequence (5'-CCACGCAGTCAACCTTCTG-3') was based on human BLT2 cDNA sequences (Hennig et al., 2008). HaCaT cells were transfected with scrambled $(50 \mu \mathrm{M})$ or BLT2 specific siRNA $(50 \mu \mathrm{M})$ using the Oligofectamine transfection reagent (Invitrogen, Carlsbad, CA) according to the manufacturer's instructions for RNA experiments. The mRNA levels of each gene were analyzed after $24 \mathrm{~h}$ by RT-PCR to evaluate interference. 


\section{Measurement of $\mathrm{LTB}_{4}$ and 12-(S) HETE by ELISA}

After UVB irradiation $\left(5 \mathrm{~mJ} / \mathrm{cm}^{2}\right), 500 \mu$ aliquots of medium were removed at the indicated times $(0,10,20,30$, and $60 \mathrm{~min}$ ) and centrifuged at $12,000 \mathrm{rpm}$ for $15 \mathrm{~min}$ at $4^{\circ} \mathrm{C}$. The supernatants were freeze-dried overnight and reconstituted with the assay buffer supplied with the $L_{T B}$ or 12(S)-HETE ELISA kits (Assay Designs, Ann Arbor, MI). $\mathrm{LTB}_{4}$ and 12(S)-HETE were measured according to the manufacturers' instructions.

\section{Measurement of ROS}

$\mathrm{HaCaT}$ cells were starved in serum-free DMEM for $24 \mathrm{~h}$ before the intracellular ROS levels were measured. Cells were treated with $1 \mu \mathrm{M}$ U75302 or $10 \mu \mathrm{M} \mathrm{LY} 255283$ for $1 \mathrm{~h}$ prior to UVB irradiation. After UVB irradiation for the indicated times, the levels of ROS were measured by FACS using Cell Quest Pro software (BD Bioscience, San Diego, CA). Before the measurement of intracellular $\mathrm{H}_{2} \mathrm{O}_{2}$, the cells were incubated for $20 \mathrm{~min}$ in the dark at $37^{\circ} \mathrm{C}$ with 20 $\mu \mathrm{M}$ 2',7'-dichlorofluorescein diacetate (Molecular Probes, Eugene, OR), an $\mathrm{H}_{2} \mathrm{O}_{2}$-sensitive fluorophore, as previously described (Woo et al., 2002).

\section{MMP-1 promoter activity}

$\mathrm{HaCaT}$ cells were transfected with $5 \mu \mathrm{g}$ of human MMP-1 luciferase reporter construct using the Lipofectamine transfection reagent (Invitrogen, Carlsbad, CA) according to the manufacturer's instructions. The MMP-1 luciferase reporter construct was kindly provided by Dr. Jin-Ho Chung (College of Medicine, Seoul National University; Kim et al., 2009). To monitor variations in cell number and transfection efficiency, $\mathrm{HaCaT}$ cells were cotransfected with $1 \mu \mathrm{g}$ of pSV40- $\beta$-galactosidase, a eukaryotic expression vector containing the Escherichia coli $\beta$-galactosidase (lacZ) structural gene under control of the SV40 promoter. At $24 \mathrm{~h}$ after transfection, the cells were starved for $24 \mathrm{~h}$ in serum-free DMEM prior to UVB irradiation. Luciferase activity was measured using a Junior luminometer (Berthold, Germany) at $24 \mathrm{~h}$ after UVB irradiation $\left(5 \mathrm{~mJ} / \mathrm{cm}^{2}\right)$. The relative fold increase in luciferase activity was calculated as previously described (Woo et al., 2005).

\section{Statistical analysis}

All experiments were performed at least twice with similar results, and representative results are shown. The results are presented as means \pm S.D. Analyses were performed with Student's $t$-test using SigmaPlot software (St. Louis, MO). Values of $P<0.05$ were considered significant. ${ }^{*} P<$ $0.05,{ }^{* *} P<0.01,{ }^{* * *} P<0.001$.

\section{Acknowledgements}

We sincerely thank Dr. Takao Shimizu (University of Tokyo, Tokyo, Japan) and Dr. Takehiko Yokomizo (University of Kyushu, Fukuoka, Japan) for the BLT2 expression plasmid and Dr. Jin-Ho Chung (College of Medicine, Seoul National University, Seoul, Korea) for the human MMP-1 reporter gene construct. This work was supported by a grant from
SRC program (Aging and Apoptosis Research Center, 2010-0001321), a General Researcher Support Project grant (2010-0008295), and a Diseases Network Research Program grant (2009-0084183) from the Ministry of Education, Science and Technology, Republic of Korea. Additionally, this work was supported by a grant of the Korea Healthcare technology Research and Development Project (A101032) from the Ministry of Health \& Welfare, Republic of Korea.

\section{References}

Brenneisen P, Sies H, Scharffetter-Kochanek K. Ultraviolet-B irradiation and matrix metalloproteinases: from induction via signaling to initial events. Ann N Y Acad Sci 2002;973:31-43

Choi JA, Kim EY, Song H, Kim C, Kim JH. Reactive oxygen species are generated through a BLT2-linked cascade in Ras-transformed cells. Free Radic Biol Med 2008;44:624-34

El-Abaseri TB, Fuhrman J, Trempus C, Shendrik I, Tennant RW, Hansen LA. Chemoprevention of UV light-induced skin tumorigenesis by inhibition of the epidermal growth factor receptor. Cancer Res 2005;65:3958-65

Faustin B, Reed JC. Sunburned skin activates inflammasomes. Trends Cell Biol 2008;18:4-8

Fisher GJ, Datta SC, Talwar HS, Wang ZQ, Varani J, Kang $\mathrm{S}$, Voorhees JJ. Molecular basis of sun-induced premature skin ageing and retinoid antagonism. Nature 1996;379: 335-9

Garmyn M, Degreef H. Suppression of UVB-induced c-fos and c-jun expression in human keratinocytes by $\mathrm{N}$-acetylcysteine. J Photochem Photobiol B 1997;37:125-30

He YY, Huang JL, Chignell CF. Delayed and sustained activation of extracellular signal-regulated kinase in human keratinocytes by UVA: implications in carcinogenesis. J Biol Chem 2004;279:53867-74

Hennig R, Osman T, Esposito I, Giese N, Rao SM, Ding XZ, Tong WG, Buchler MW, Yokomizo T, Friess H, Adrian TE. BLT2 is expressed in PanINs, IPMNs, pancreatic cancer and stimulates tumour cell proliferation. Br J Cancer 2008;99: 1064-73

Hruza LL, Pentland AP. Mechanisms of UV-induced inflammation. J Invest Dermatol 1993;100:35S-41S

lizuka Y, Yokomizo T, Terawaki K, Komine M, Tamaki K, Shimizu T. Characterization of a mouse second leukotriene B4 receptor, mBLT2: BLT2-dependent ERK activation and cell migration of primary mouse keratinocytes. $\mathrm{J}$ Biol Chem 2005;280:24816-23

Isoherranen K, Punnonen K, Jansen C, Uotila P. Ultraviolet irradiation induces cyclooxygenase-2 expression in keratinocytes. Br J Dermatol 1999;140:1017-22

Jenkins G. Molecular mechanisms of skin ageing. Mech Ageing Dev 2002;123:801-10

Kang S, Chung JH, Lee JH, Fisher GJ, Wan YS, Duell EA, Voorhees JJ. Topical $\mathrm{N}$-acetyl cysteine and genistein prevent 
ultraviolet-light-induced signaling that leads to photoaging in human skin in vivo. J Invest Dermatol 2003;120:835-41

Katiyar SK, Matsui MS, Elmets CA, Mukhtar H. Polyphenolic antioxidant (-)-epigallocatechin-3-gallate from green tea reduces UVB-induced inflammatory responses and infiltration of leukocytes in human skin. Photochem Photobiol 1999;69:148-53

Kim HH, Shin CM, Park CH, Kim KH, Cho KH, Eun HC, Chung $\mathrm{JH}$. Eicosapentaenoic acid inhibits UV-induced MMP-1 expression in human dermal fibroblasts. J Lipid Res 2005; 46:1712-20

Kim HJ, Kim DK, Kim H, Koh JY, Kim KM, Noh MS, Lee S, Kim S, Park SH, Kim JJ, Kim SY, Lee CH. Involvement of the $\mathrm{BLT} 2$ receptor in the itch-associated scratching induced by 12-(S)-lipoxygenase products in ICR mice. Br J Pharmacol 2008;154:1073-8

Kim MK, Shin JM, Eun HC, Chung JH. The role of p300 histone acetyltransferase in UV-induced histone modifications and MMP-1 gene transcription. PLoS One 2009;4: e4864

Lee ER, Kang YJ, Kim JH, Lee HT, Cho SG. Modulation of apoptosis in $\mathrm{HaCaT}$ keratinocytes via differential regulation of ERK signaling pathway by flavonoids. J Biol Chem 2005; 280:31498-507

Matrisian LM. Matrix metalloproteinase gene expression. Ann N Y Acad Sci 1994;732:42-50

Matsumura Y, Ananthaswamy HN. Toxic effects of ultraviolet radiation on the skin. Toxicol Appl Pharmacol 2004;195: 298-308

Pillai S, Oresajo C, Hayward J. Ultraviolet radiation and skin aging: roles of reactive oxygen species, inflammation and protease activation, and strategies for prevention of inflammation-induced matrix degradation - a review. Int J Cosmet Sci 2005;27:17-34

Rhodes LE, Gledhill K, Masoodi M, Haylett AK, Brownrigg M, Thody AJ, Tobin DJ, Nicolaou A. The sunburn response in human skin is characterized by sequential eicosanoid profiles that may mediate its early and late phases. FASEB $J$ 2009;23:3947-56

Ryu HC, Kim C, Kim JY, Chung JH, Kim JH. UVB radiation induces apoptosis in keratinocytes by activating a pathway linked to "BLT2-reactive oxygen species". J Invest Dermatol 2010;130:1095-106

Saliou C, Kitazawa M, McLaughlin L, Yang JP, Lodge JK, Tetsuka T, Iwasaki K, Cillard J, Okamoto T, Packer L. Antioxidants modulate acute solar ultraviolet radiationinduced NF-kappa-B activation in a human keratinocyte cell line. Free Radic Biol Med 1999;26:174-83

Seo JY, Kim EK, Lee SH, Park KC, Kim KH, Eun HC, Chung $\mathrm{JH}$. Enhanced expression of cylooxygenase-2 by UV in aged human skin in vivo. Mech Ageing Dev 2003;124:903-10

Shim JS, Han YS, Hwang JK. The effect of 4-hydroxypanduratin $A$ on the mitogen-activated protein kinase-dependent activation of matrix metalloproteinase-1 expression in human skin fibroblasts. J Dermatol Sci 2009;53:129-34
Shin MH, Moon YJ, Seo JE, Lee Y, Kim KH, Chung JH. Reactive oxygen species produced by NADPH oxidase, xanthine oxidase, and mitochondrial electron transport system mediate heat shock-induced MMP-1 and MMP-9 expression. Free Radic Biol Med 2008;44:635-45

Sternlicht MD, Werb Z. How matrix metalloproteinases regulate cell behavior. Annu Rev Cell Dev Biol 2001;17: 463-516

Tager AM, Luster AD. BLT1 and BLT2: the leukotriene B(4) receptors. Prostaglandins Leukot Essent Fatty Acids 2003; 69:123-34

Vayalil PK, Mittal A, Hara Y, Elmets CA, Katiyar SK. Green tea polyphenols prevent ultraviolet light-induced oxidative damage and matrix metalloproteinases expression in mouse skin. J Invest Dermatol 2004;122:1480-7

Wertz K, Seifert N, Hunziker PB, Riss G, Wyss A, Lankin C, Goralczyk R. Beta-carotene inhibits UVA-induced matrix metalloprotease 1 and 10 expression in keratinocytes by a singlet oxygen-dependent mechanism. Free Radic Biol Med 2004;37:654-70

Whitmarsh AJ, Davis RJ. Transcription factor AP-1 regulation by mitogen-activated protein kinase signal transduction pathways. J Mol Med 1996;74:589-607

Woo CH, You HJ, Cho SH, Eom YW, Chun JS, Yoo YJ, Kim $\mathrm{JH}$. Leukotriene $\mathrm{B}(4)$ stimulates Rac-ERK cascade to generate reactive oxygen species that mediates chemotaxis. J Biol Chem 2002;277:8572-8

Woo CH, Yoo MH, You HJ, Cho SH, Mun YC, Seong CM, Kim $\mathrm{JH}$. Transepithelial migration of neutrophils in response to leukotriene B4 is mediated by a reactive oxygen species-extracellular signal-regulated kinase-linked cascade. J Immunol 2003;170:6273-9

Woo $\mathrm{CH}$, Lim JH, Kim JH. VCAM-1 upregulation via PKCdelta-p38 kinase-linked cascade mediates the TNFalpha-induced leukocyte adhesion and emigration in the lung airway epithelium. Am J Physiol Lung Cell Mol Physiol 2005;288:L307-16

Yan Y, Wang B, Zuo YG, Qu T. Inhibitory effects of mizolastine on ultraviolet B-induced leukotriene B4 production and 5-lipoxygenase expression in normal human dermal fibroblasts in vitro. Photochem Photobiol 2006;82:665-9

Yang B, Ji C, Kang J, Chen W, Bi Z, Wan Y. Trans-Zeatin inhibits UVB-induced matrix metalloproteinase-1 expression via MAP kinase signaling in human skin fibroblasts. Int J Mol Med 2009;23:555-60

Yoshizumi M, Nakamura T, Kato M, Ishioka T, Kozawa K, Wakamatsu K, Kimura H. Release of cytokines/chemokines and cell death in UVB-irradiated human keratinocytes, HaCaT. Cell Biol Int 2008;32:1405-11

Zaid MA, Afaq F, Syed DN, Dreher M, Mukhtar H. Inhibition of UVB-mediated oxidative stress and markers of photoaging in immortalized $\mathrm{HaCaT}$ keratinocytes by pomegranate polyphenol extract POMx. Photochem Photobiol 2007;83: 882-8 